\title{
Bronchogenic glomangiomyoma with local intravenous infiltration
}

\author{
M. Katabami ${ }^{\star,+}$, K. Okamoto ${ }^{\#,+}$, K. Ito ${ }^{\oplus}$, K. Kimura* and H. Kaji*
}

ABSTRACT: Most glomus tumours occur in the dermis and subcutaneous tissues. Lung glomus tumours are quite rare.

The current authors present the first reported case of a lung-derived glomangiomyoma, the rarest variant of glomus tumour.

A 56-yr-old female was admitted with haemoptysis. Chest computed tomography showed an $\sim 5$-cm-diameter mass in the right lower lobe with mucoid impaction. After a right lower lobectomy, a diagnosis of glomangiomyoma was made.

The tumour had grown endobronchially and its maximal diameter was $5.5 \mathrm{~cm}$. Although cytologically benign, glomus tumour cells had visibly infiltrated neighbouring vessels. These results suggest that a bronchogenic glomangiomyoma has a low-grade malignancy potential and warrants close follow-up.

KEYWORDS: Bronchogenic glomangiomyoma, glomus tumour, intravenous infiltration, low-grade malignancy

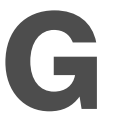
lomus tumours are presumed to originate from the modified smooth muscle cells of glomus bodies, which are thermoregulating arteriovenous anastomoses. Glomus tumours are mostly benign neoplasms arising from the distal parts of the dermis and subcutaneous tissues, where there is an abundance of glomus bodies. However, glomus tumours occasionally occur in the viscera, where glomus bodies are believed to be sparse or even absent. Lung glomus tumours are quite rare, with only 16 cases reported so far in the English-language literature. The current authors present the first case of a lungderived glomangiomyoma, the rarest variant of glomus tumour, and highlight its unusual clinicopathological aspects.

\section{CASE REPORT}

A 56-yr-old female smoker presented with sudden haemoptysis and was admitted to hospital (Iwamizawa Rosai Hospital, Iwamizawa, Japan). Over the previous 2 yrs, the patient had been followed for bronchiectasis at a nearby clinic, during which time she had been asymptomatic. Admission chest computed tomography (CT) scans showed an $\sim 5$-cm-diameter, wellcircumscribed lobular mass extending downward into the right lower lobe (fig. 1a), along with several irregularly shaped opacities distal to the mass (fig. 1b). The opacities appeared to contain some airspaces (fig. 1b). Physical and radiography examinations did not reveal any other primary tumours elsewhere in the body. Laboratory tests were all within the normal smooth-surfaced, ruddy grey mass that bled easily and occupied the entrance to the rightstem basal bronchus (fig. $1 \mathrm{c}$ and $\mathrm{d}$ ). This finding was suggestive of a bronchial carcinoid tumour, but biopsy specimens were not diagnostic. Since it seemed likely that the patient's haemoptysis was originating from this large haemorrhagic mass, a video-assisted right lower lobectomy was carried out.

Macroscopically, the grey-brown solid mass had grown endobronchially to a size of $5.5 \times 4.4 \times$ $3.4 \mathrm{~cm}$. The mass occupied the area from the stem basal bronchial lumen to the subsegmental lumens of the right lower bronchus (fig. 2a and $b$ ). Solid nodules were detected inside the large veins adjacent to the main mass, suggesting intravenous infiltration of the lesions (fig. 2a). Mucoid impaction was found in the dilated bronchi around the periphery of the mass, corresponding to the distal opacities seen on chest CT (figs $1 \mathrm{~b}$ and $2 \mathrm{c}$ ). The mass was composed of almost equal amounts of three range. Bronchoscopic examination revealed a

\section{AFFILIATIONS}

Depts of *Internal Medicine

\#Pathology, and

"Surgery, Iwamizawa Rosai Hospital, Iwamizawa, Japan.

${ }^{+}$Authors contributed equally to the study.

\section{CORRESPONDENCE}

M. Katabami

Dept of Internal Medicine Iwamizawa Rosai Hospital 4-Jo Higashi 16-Chome Iwamizawa 068-0004 Japan

Fax: 81126221304

E-mail: katabamm@sea.plala.or.jp

Received:

January 062006

Accepted after revision:

May 182006 

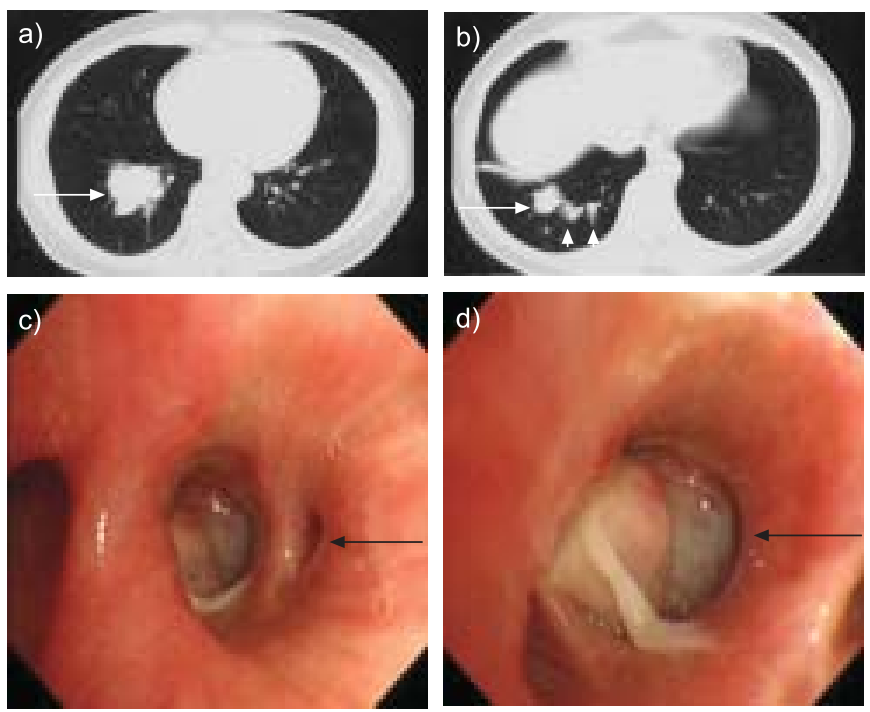

FIGURE 1. $a$ and $b)$ Chest computed tomography scans showing an $\sim 5-\mathrm{cm}$ diameter well-circumscribed lobular mass (arrows) extending downward into the right lower lobe, with opacities (white arrowheads) distal to the mass. c and d) Bronchoscopic examination shows a smooth-surfaced haemorrhagic mass (arrows) occupying the right lower lobe bronchus.
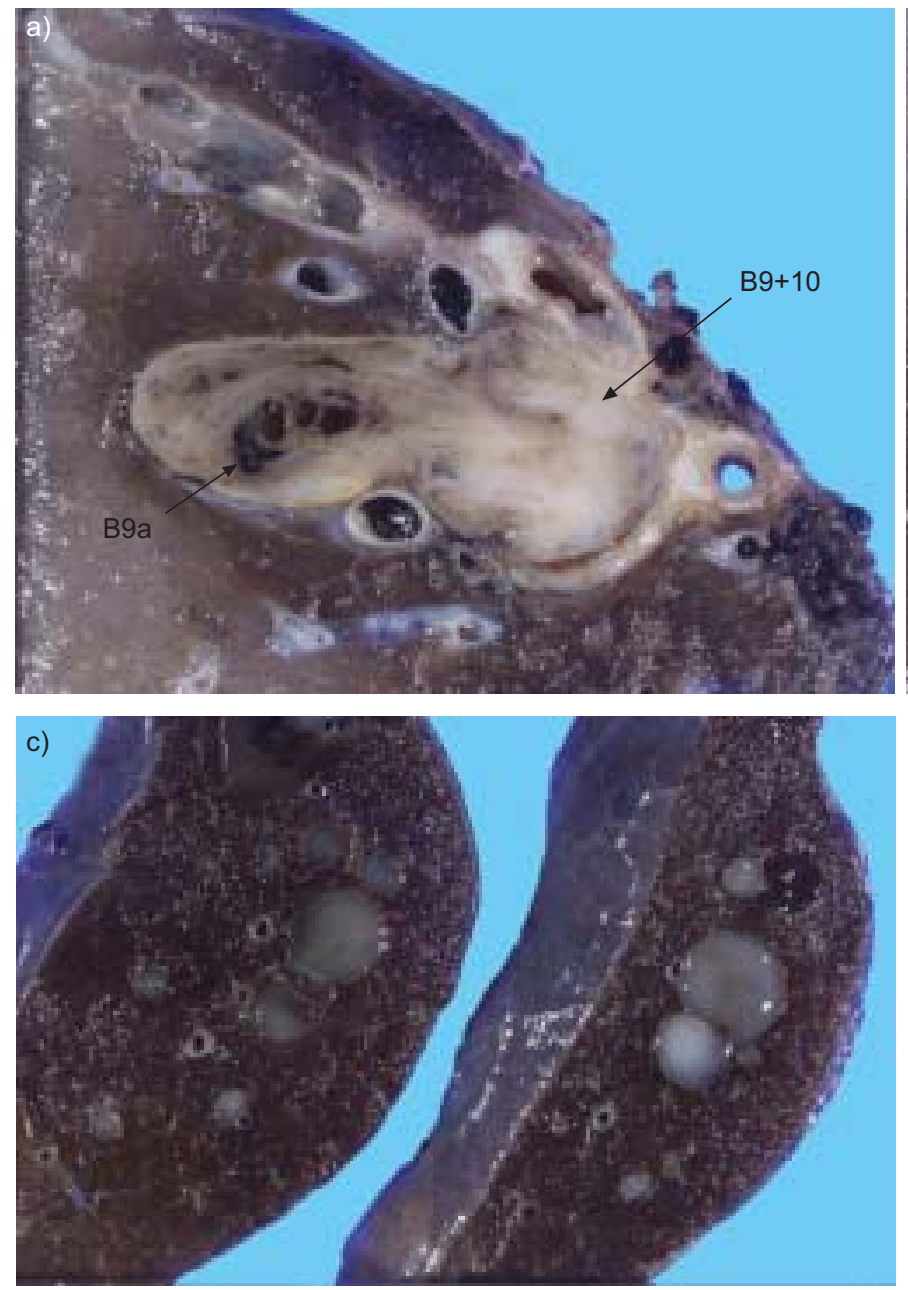

distinct components (uniform round epithelioid cells, spindle leiomyomatous cells and intermingled blood vessels), as shown by the magnifying-glass view (fig. 3).

Microscopically, round cells were seen that characteristically had a clear eosinophilic cytoplasm with diffuse fine chromatin (fig. 4a). These features of the round cells are very reminiscent of glomus tumours that usually arise from the dermis. Spindle cells were found to be arranged either in bundles or in a more irregular manner (fig. 4b). These arrangements of spindle cells were seen side by side with clusters of round cells (fig. 4c). Abundant branching vessels were intermingled with the solid tumour components (fig. 4d). Immunohistochemical study revealed that the tumour cells were positive for $\alpha$-smooth muscle actin (1:50; Dako, Glostrup, Denmark; fig. 5a), musclespecific actin (using the mouse monoclonal antibody HHF-35; 1:50; Nichirei, Tokyo, Japan; fig. 5b), vimentin (1:100; Dako), and weakly positive for desmin (1:50; Dako; fig. 5c), and were negative for CD56 (1:100; Novocastra, Newcastle-upon-Tyne, UK), S-100 protein (1:500; Dako), chromogranin (1:50; MBL, Woburn, MA, USA) and CD34 (1:50; Dako; data not shown). These findings supported the definite diagnosis of a glomus tumour. Furthermore, given that both spindle cells and intermingled blood vessels were more frequently seen than the typical round glomus cells, the present case was diagnosed

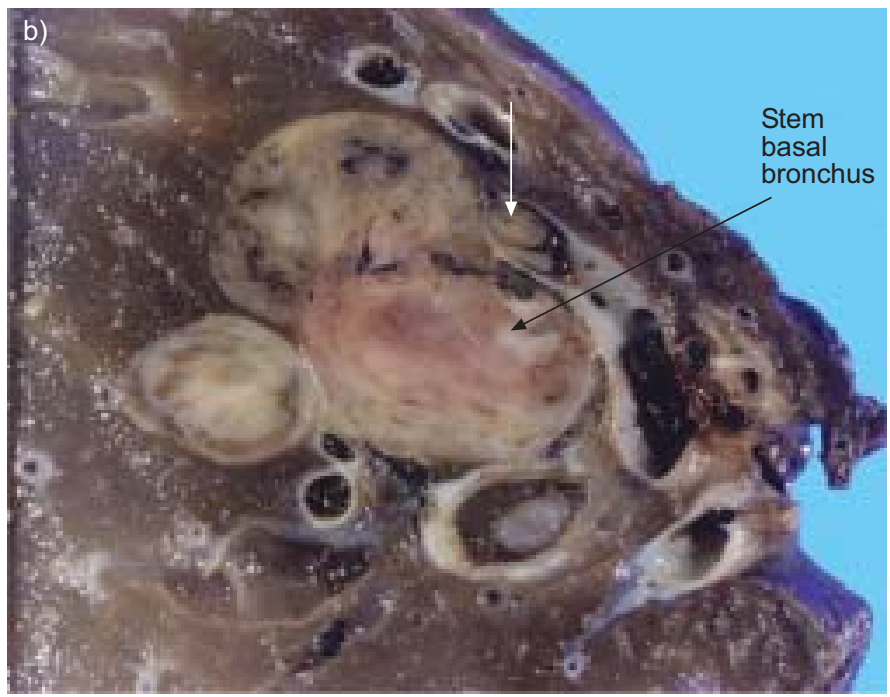

FIGURE 2. a, b) The $5.5 \times 4.4 \times 3.4-\mathrm{cm}$ grey-brown solid mass grew endobronchially (arrow) and dilated the area from the stem basal bronchus to the sub-segmental lumens (B9, B10 and B9a) of the right lower bronchus (arrows). b) A solid nodule (white arrow) detected inside a large vein adjacent to the main mass. c) Pronounced mucoid impaction was found around the periphery of the mass. 

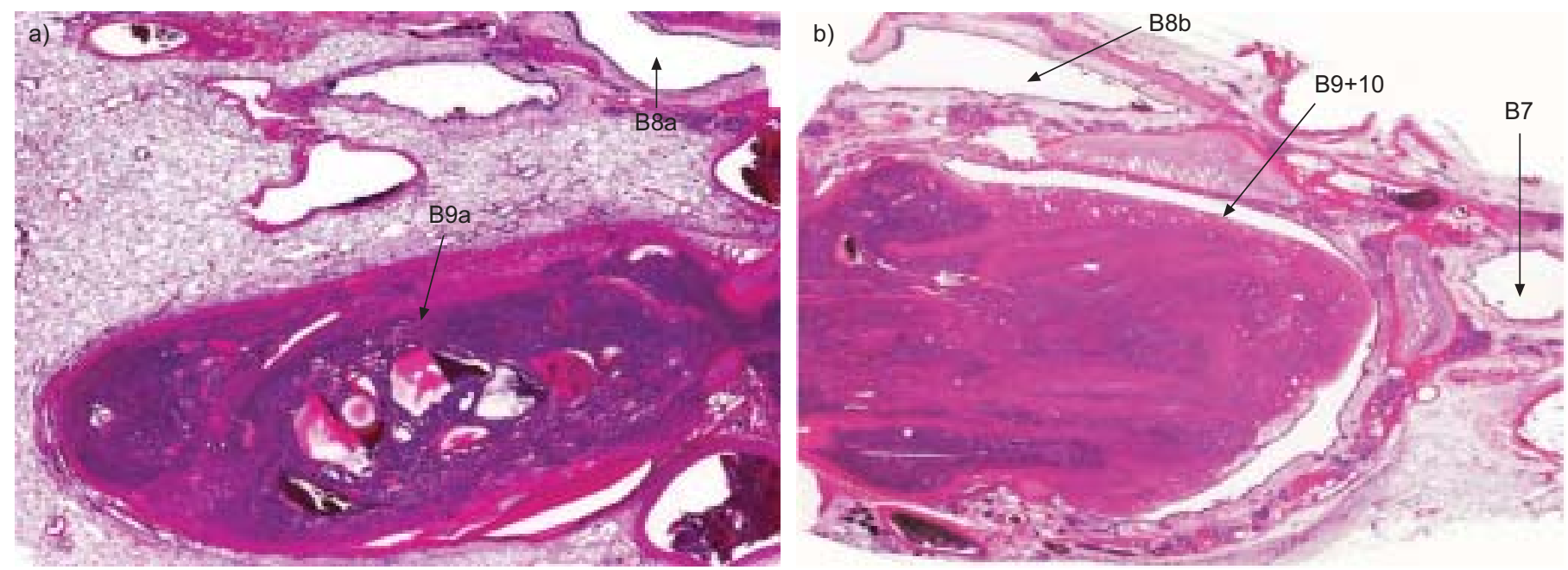

FIGURE 3. Magnifying-glass appearance of the tumour, showing almost equal amounts of three distinct cellular components: a) angiomatous lesions with abundant intermingled blood vessels; and b) lesions with uniform round epitheloid cells (blue-stained areas), and lesions with spindle leiomyomatous cells (red-stained areas).
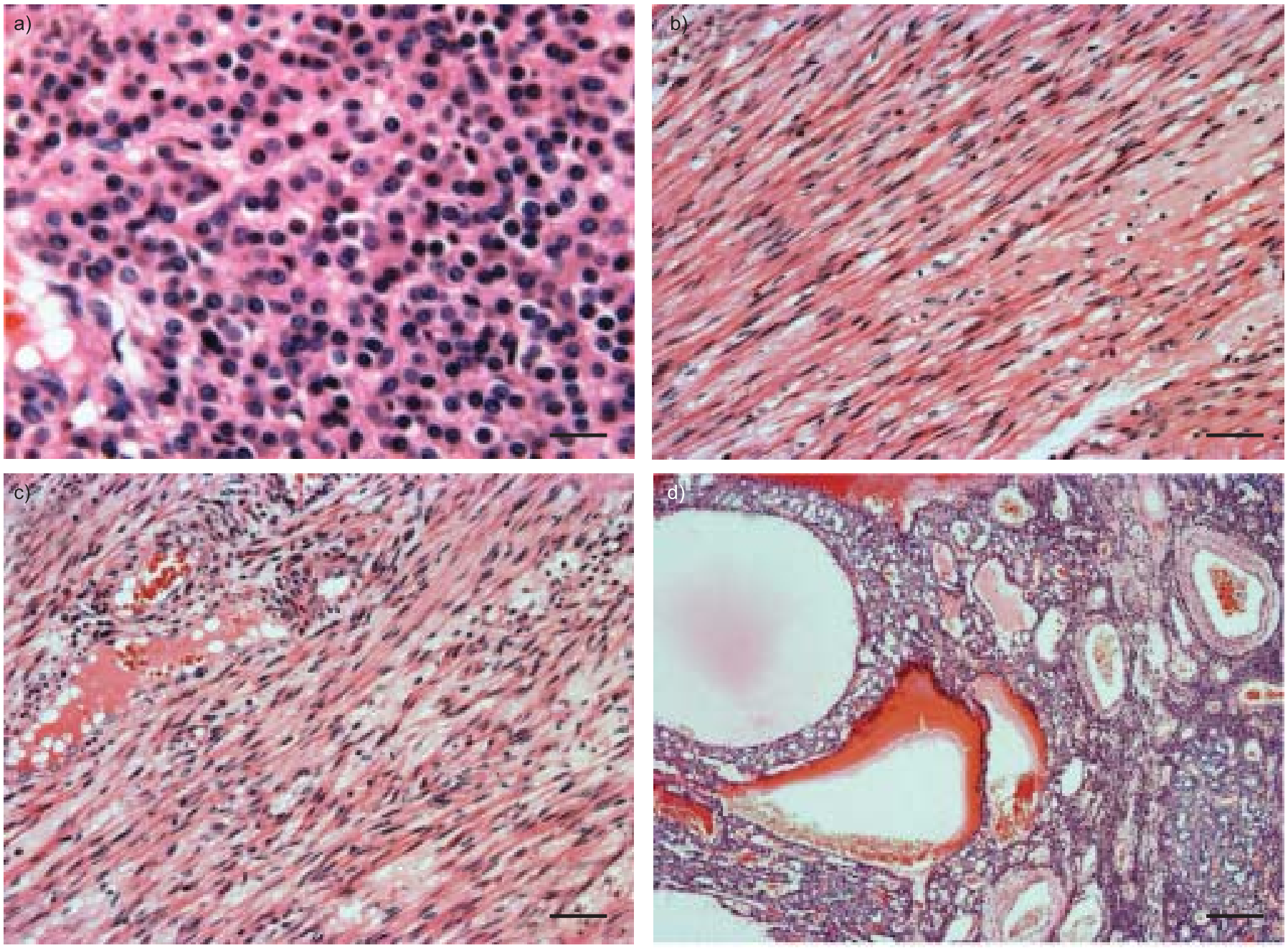

FIGURE 4. a) Uniform round cells with eosinophilic cytoplasm. b) Spindle cells arranged in bundles or in a more irregular manner. c) The components of the spindle cells are seen merging with those of the uniform round cells. d) Abundant branching vessels intermingled with solid tumour components. Haematoxylin and eosin stain was used. Scale bars $=25 \mu \mathrm{m}(\mathrm{a}), 50 \mu \mathrm{m}(\mathrm{b}$ and $\mathrm{c}$ ) and $0.25 \mathrm{~mm}(\mathrm{~d})$. 

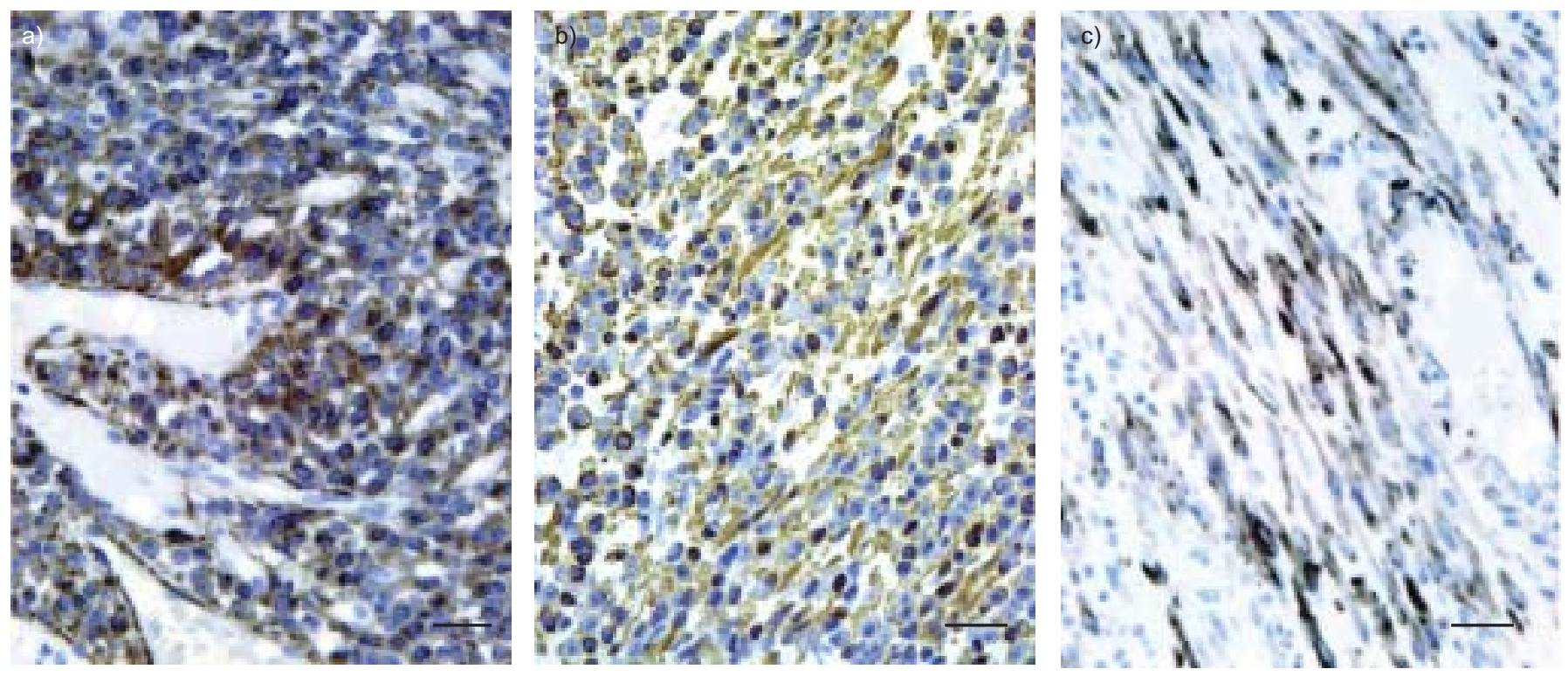

FIGURE 5. Positive immunoreactivity of a) $\alpha$-smooth muscle actin (SMA; 1:50; Dako, Glostrup, Denmark), b) muscle-specific actin (using mouse monoclonal antibody HHF-35; 1:50; Nichirei, Tokyo, Japan) and c) desmin (1:50; Dako). Scale bars $=25 \mu \mathrm{m}$.
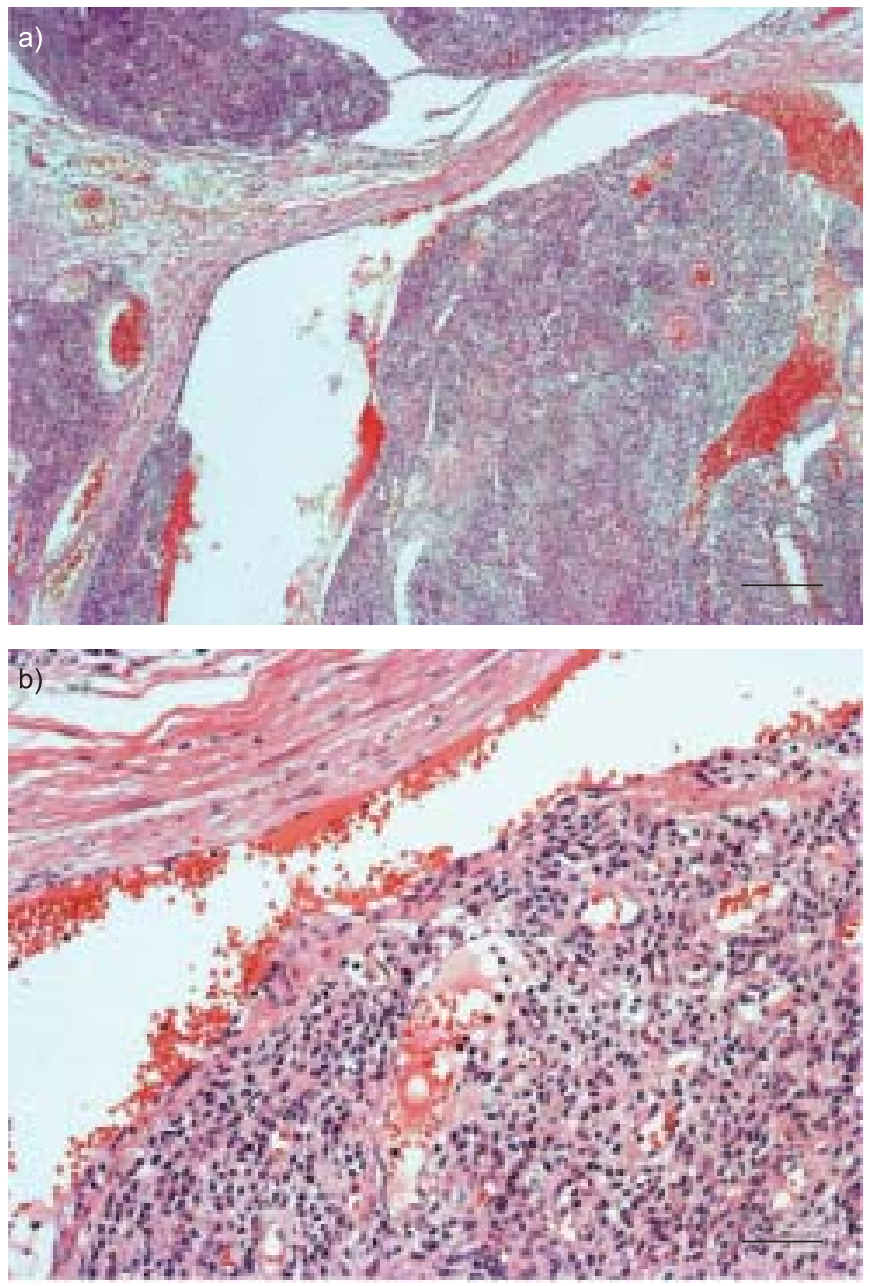

FIGURE 6. Tumour cells showing intravenous infiltration in a) low- and b) highpower view. Haematoxylin and eosin stain was used. Scale bars $=0.5 \mathrm{~mm}$ (a) and $0.1 \mathrm{~mm}(\mathrm{~b})$. as a glomangiomyoma, the rarest variant of glomus tumour. Cellular atypia and necrosis were only rarely seen in this tumour. There was no infiltration of tumour cells into the lymph nodes. However, tumour cells infiltrated discernibly into the adjacent vessels and proliferated inside the vessels (figs $2 \mathrm{a}$ and 6). The tumour extended to the contiguous pulmonary parenchyma. One year after surgical resection of the tumour, the patient has remained stable.

\section{DISCUSSION}

A lung carcinoid tumour typically presents as a proximal smooth haemorrhagic mass prone to cause haemoptysis, as occurred in the current case [1]. Carcinoid tumours arise much more frequently in the lung than glomus tumours. Mucoid impaction has also been documented to occur in lung carcinoid tumours [2]. In the current patient, carcinoid tumour was the initial pre-operative diagnosis. Carcinoid tumour cells have a neuroendocrine origin, whereas glomus tumours are derived from modified smooth muscle cells and are almost always positive for $\alpha$-smooth muscle actin (SMA), HHF-35 and vimentin. In the present case, the tumour was negative for neuroendocrine markers (CD56 and S-100 protein), which allowed the possibility of carcinoid tumour to be excluded. Haemangiopericytoma may be another important differential diagnosis, since its pathological features are often confused with glomus tumours, particularly with those glomus tumours that possess copious spindle cells. However, in the current case, a large number of epithelioid round cells, typical of glomus cells, were present, and this allowed the glomus tumour to be distinguished readily from a haemangiopericytoma. Furthermore, haemangiopericytomas are frequently positive for CD34 and negative for $\alpha$-SMA and desmin, whereas in the present case the opposite findings were noted, thus making a diagnosis of haemangiopericytoma highly unlikely $[3,4]$.

Glomus tumours can be subdivided pathologically into glomus tumour proper, glomangioma and glomangiomyoma, based 
on the relative predominance of the three major constituents: round glomus cells in glomus tumour proper; blood vessels in glomangioma; and spindle cells in glomangiomyoma. Glomus tumour proper is the most common, followed by glomangioma. Glomangiomyoma is the rarest variant with a frequency as low as $8 \%$ of all glomus tumours [5]. This case was diagnosed as a glomangiomyoma, as it had a pronounced spindle-cell component that existed side by side with typical round glomus cells. The abundance of these transitional zones is a distinct feature of glomangiomyoma [5]. To the best of the authors' knowledge, the current case is the first lung glomangiomyoma ever reported.

Another striking feature that was noted in the current case was the presence of intravenous infiltration by the glomus tumour cells. A recent re-evaluation of atypical and malignant glomus tumours did not classify vascular space involvement per se as a malignant finding [6]. However, this re-evaluation was based mainly on skin or subcutaneous glomus tumours. Thus, it may be premature to apply this concept to pulmonary glomus tumours, given the clinicopathological differences between extra-visceral and visceral glomus tumours [7, 8]. A recent report noted that a case of gastric glomus tumour accompanied by intravascular infiltration with only mild atypia was found to be malignant 33 months after resection [7]. The abundance of spindle-cell foci and a tumour size $>5 \mathrm{~cm}$ may also be potential risk factors for malignancy in visceral glomus tumours $[7,8]$. These factors were all present in this case. In particular, the tumour's maximal diameter was $5.5 \mathrm{~cm}$, which is by far the largest of all previously reported glomus tumours with endobronchial growth (including one malignancy), whose diameters ranged $0.7-2.5 \mathrm{~cm}$ [8-12]. Based on these findings, the current case of bronchogenic glomangiomyoma was considered to have the potential for low-grade malignancy.

In conclusion, the present authors have described the first glomangiomyoma (the rarest variant of glomus tumour) ever reported in the lung. Since the tumour presented as an endobronchial smooth haemorrhagic mass, carcinoid tumour was first considered in the differential diagnosis. After surgical resection, a diagnosis of glomangiomyoma was made, based on histopathology and immunohistochemistry. Notably, since this tumour was $>5 \mathrm{~cm}$ in size and was accompanied by intravenous infiltration, there is a potential for low-grade malignancy. Therefore, close follow-up is warranted even after curative resection.

\section{ACKNOWLEDGEMENTS}

The authors would like to thank R. Sato, I. Sakai, M. Asada and M. Hashimoto for their clinical support and suggestions.

\section{REFERENCES}

1 Hage $\mathrm{R}$, de la Riviere AB, Seldenrijk CA, van den Bosch JM. Update in pulmonary carcinoid tumors. Ann Surg Oncol 2003; 10: 697-704.

2 Jeung MY, Gasser B, Gangi A, et al. Bronchial carcinoid tumors of the thorax: spectrum of radiologic findings. Radiographics 2002; 22: 351-365.

3 Nappi O, Ritter JH, Pettinato G, Wick MR. Hemangiopericytoma: histopathological pattern or clinicopathologic entity? Semin Diagn Pathol 1995; 12: 221-232.

4 Porter PL, Bigler SA, McNutt M, Gown AM. The immunophenotype of hemangiopericytomas and glomus tumors, with special reference to muscle protein expression: an immunohistochemical study and review of the literature. Mod Pathol 1991; 4: 46-52.

5 Enzinger FM, Weiss SW. Perivascular tumors. In: Enzinger FM, Weiss SW, eds. Soft Tissue Tumors. 4th Edn. St Louis, Mosby, 2001; pp. 985-1001.

6 Folpe AL, Fanburg-Smith JC, Miettinen M, Weiss SW. Atypical and malignant glomus tumors: analysis of 52 cases, with a proposal for the reclassification of glomus tumors. Am J Surg Pathol 2001; 25: 1-12.

7 Miettinen M, Paal E, Lasota J, Sobin LH. Gastrointestinal glomus tumors: a clinicopathologic, immunohistochemical, and molecular genetic study of 32 cases. Am J Surg Pathol 2002; 26: 301-311.

8 Zhang Y, England DM. Primary pulmonary glomus tumor with contiguous spread to a peribronchial lymph node. Ann Diagn Pathol 2003; 7: 245-248.

9 Gaertner EM, Steinberg DM, Huber M, et al. Pulmonary and mediastinal glomus tumors: report of five cases including a pulmonary glomangiosarcoma: a clinicopathologic study with literature review. Am J Surg Pathol 2000; 24: 1105-1114.

10 Oizumi S, Kon Y, Ishida $\mathrm{T}$, et al. A rare case of bronchial glomus tumor. Respiration 2001; 68: 95-98.

11 Yilmaz A, Bayramgurler B, Aksoy F, Tuncer LY, Selvi A, Uzman O. Pulmonary glomus tumour: a case initially diagnosed as carcinoid tumour. Respirology 2002; 7: 369-371.

12 Hishida T, Hasegawa $\mathrm{T}$, Asamura $\mathrm{H}$, et al. Malignant glomus tumor of the lung. Pathol Int 2003; 53: 632-636. 